\title{
San Juan single-well seismic data analysis and modeling study
}

Chunling $W u^{*}$ and Jerry M. Harris, Stanford University

Thomas M. Daley and Ernest L. Majer, Lawrence Berkeley National Laboratory

\section{Summary}

We analyze single-well seismic data from the San Juan Basin in Northwest New Mexico. The consistently observable events are tube-waves: direct, reflected and multiple tube-waves can be explained by the formation properties and survey geometry except for an anomalous zone with low velocity, high amplitude and horizontal polarization.

To aid the data analysis, forward modeling using a variable-grid finite-difference parallel code is performed. The numerical result confirms the identified events in the field observations.

\section{Introduction}

Sponsored by the U.S. Dept. of Energy, a large study on fracture quantification in naturally fractured gas reservoirs has been undertaken (Majer et al., 2001). As part of the project, field data at different scales (logs, single-well, VSP, and surface seismic) were collected in the San Juan Basin in Northwest New Mexico (Majer et al., 2002). We are conducting the single-well seismic data analysis and modeling study.

In single-well field data, tube-waves are the consistently observable events, though weak direct S-waves and tubewave to body-wave conversions at interfaces can be seen on some sensors. Here we present the analysis on (1) the generally visible events; and (2) an anomalous zone with low velocity and high amplitude. We also present a numerical modeling result that confirms the data analysis.

\section{Survey information}

For the San Juan single-well experiments, two surveys were attempted: one with the orbital vibrator source and 3-C (3-component) geophone receiver; the other with a high frequency piezoelectric source and hydrophone receiver. Figure 1 shows the equipment geometry. We are focusing on the orbital vibrator data analysis since only limited piezoelectric data were acquired.

For the orbital vibrator survey, the source depth ranges from 4245 to $4900 \mathrm{ft}$ at $5 \mathrm{ft}$ intervals. There are four $3-\mathrm{C}$ (H1, H2 and V) receivers per shot at offsets $87,97,107$, and $117 \mathrm{ft}$. The orbital source with clockwise and counterclockwise rotations at each location is decomposed into two orthogonally horizontal components ( $\mathrm{H} 1$ and $\mathrm{H} 2$ ); this was performed at LBNL. Therefore, six components are recorded for each source depth. Shot gathers have one source depth for four $3-\mathrm{C}$ receivers. Common offset

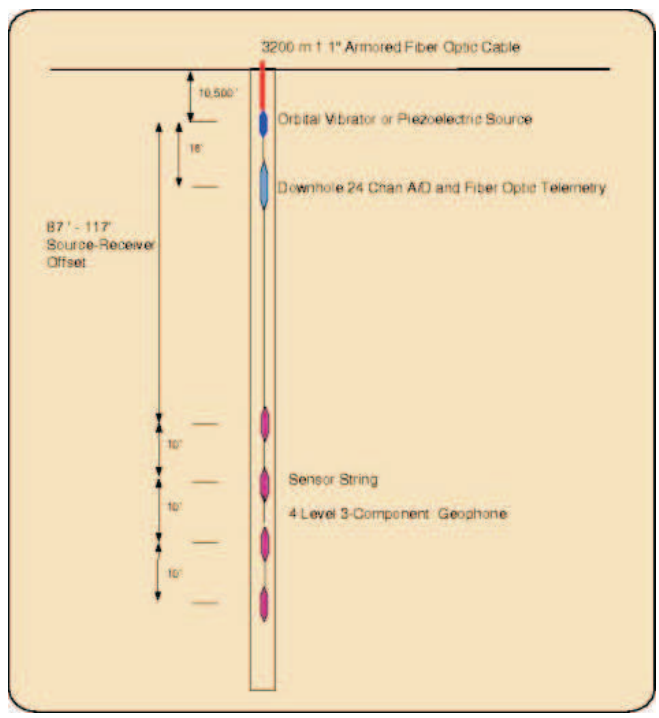

Fig. 1: Single-well equipment geometry.

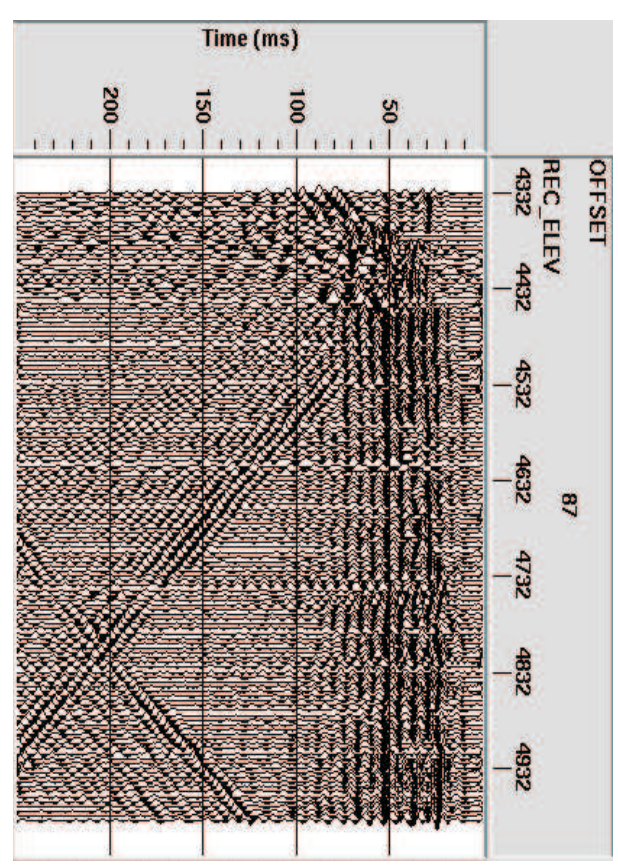

Fig. 2: One component (source $\mathrm{H} 2$, receiver $\mathrm{V}$ ) of the common offset gather of channel1 (offset $87 \mathrm{ft}$ ) with AGC. 


\section{Single-well seismic data analysis and modeling}

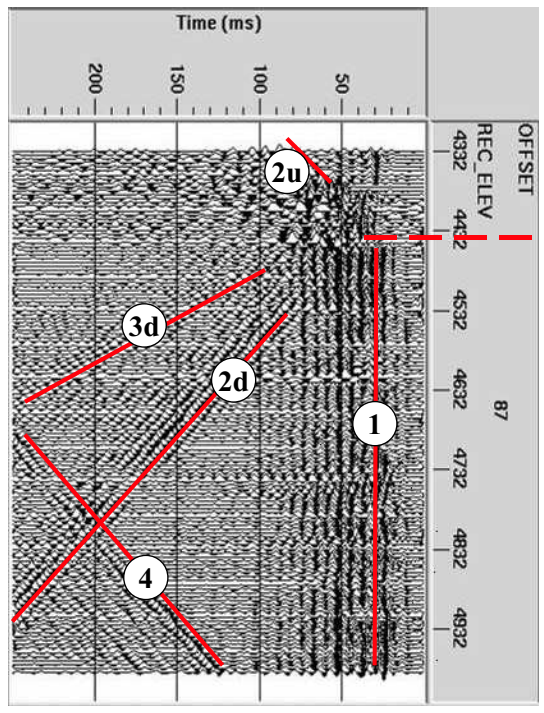

(a)

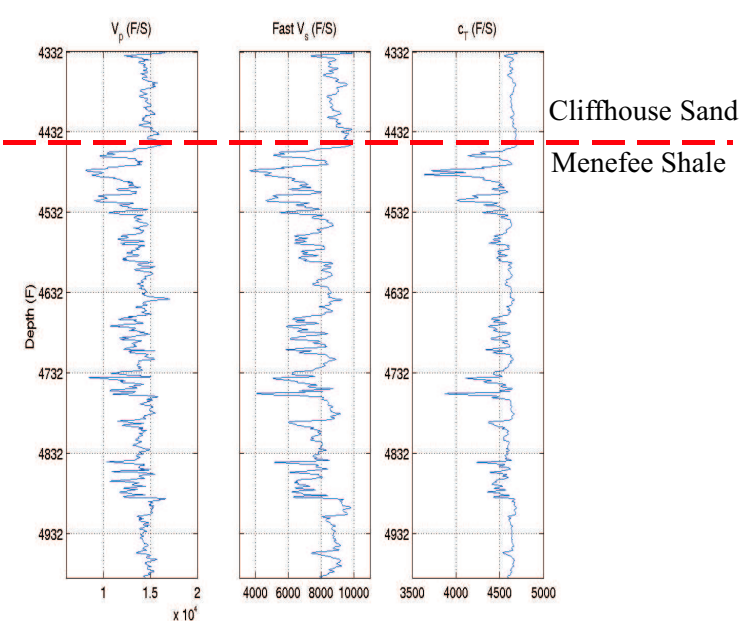

(b)

Fig. 3: (a) Generally visible events in the field data; (b) Sonic logs (P-and fast S-waves) and the estimated low frequency tube-wave velocity from logs. Velocities are used to predict the arrival times to identify the events in the data: 1 - direct tube-wave; $2 \mathrm{u} / 2 \mathrm{~d}-$ up/down-going interface reflections; $3 \mathrm{~d}$ - down-going multiple reflections; 4 - reflected tube-wave from the well plug.

gathers have a single source-receiver pair for all source depths. We use common offset gathers for data analysis since they have the whole depth coverage.

\section{Field data analysis}

Figure 2 shows one component (source $\mathrm{H} 2$, receiver V) of the common offset gather of channel1 (offset $87 \mathrm{ft}$ ). Direct and reflected waves are generally visible. A low velocity zone between depth $4387-4442 \mathrm{ft}$ is observed. We now present analysis of these.

\section{Events analysis}

Logs and survey geometry can help us to identify the events observed in the field data (Figure 3a).

Figure $3 \mathrm{~b}$ shows sonic logs ( $\mathrm{P}$ - and fast $\mathrm{S}$-waves) and the estimated low frequency $(<1000 \mathrm{~Hz})$ tube-wave velocity (White, 1983). Using these velocities, we predict the arrival times of the direct $\mathrm{P}_{-}, \mathrm{S}$ - and tube-waves which indicate that the consistently observable direct arrivals (event 1) in the field data are tube-waves, though weak $\mathrm{S}$-waves are visible on some sensors. Primary tube-wave reflections, events $2 \mathrm{u} / 2 \mathrm{~d}$ and 4 , are from the $\mathrm{C} / \mathrm{M}$ interface (Cliffhouse sand/Menefee shale interface) and the well plug, respectively. Event $3 \mathrm{~d}$ is due to multiple reflections between the tool and the $\mathrm{C} / \mathrm{M}$ interface.

Figure 4 schematically illustrates the wave propagation paths (Figure 4a) and associated arrivals on the seismogram (Figure 4b). Except the direct tube-waves (1) and the well plug reflections (4), the $\mathrm{C} / \mathrm{M}$ interface reflections and multiples are affected by the tool position. When the tool is above the interface, there are up-going primary reflections from the interface $(2 \mathrm{u})$ and multiple reflections between the tool and the interface $(3 \mathrm{u})$. When the tool is across the interface (the source is above and the receiver is below the interface), neither primary nor multiple reflections will be observed (dash lines in Figure $4 \mathrm{~b}$ ). When the tool is below the interface, there are down-going interface reflections (2d) and multiple reflections (3d).

For a common offset gather, slopes of events on the seismogram are their apparent velocities. Using the ray paths for source/receiver at two depths, we get the formulation to calculate the true velocities of the events in Figure $4 \mathrm{~b}$ :

$$
\begin{aligned}
c_{1} & =\mathrm{S}-\mathrm{R} \text { offset } / \Delta t \\
c_{2 u} & =c_{2 d}=c_{4}=2 * \Delta h / \Delta t=2 * c^{\prime} \\
c_{3 u} & =c_{3 d}=4 * \Delta h / \Delta t=4 * c^{\prime},
\end{aligned}
$$

where $c^{\prime}=\Delta h / \Delta t$, called apparent velocity, is the slope of the event on the seismogram.

Figure 5 shows the apparent and true velocities of the identified events in the field data. We can see these events have almost the same true velocities that confirm they are all tube-waves.

\section{Anomalous zone analysis}

In the field seismogram (Figure 2), for receiver depths between $4387-4442 \mathrm{ft}$, the observed direct wave has a very low velocity. True amplitude display (Figure 6 ) shows the event has a very high amplitude. The following is the analysis of this anomalous zone.

In figure 3 , we can see the anomalous zone is right above 


\section{Single-well seismic data analysis and modeling}

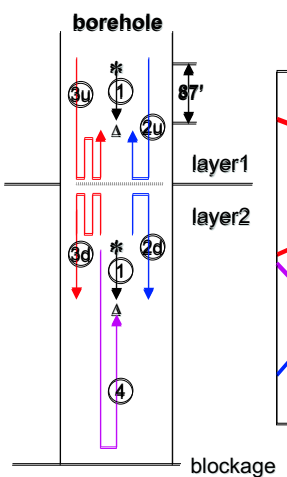

(a)

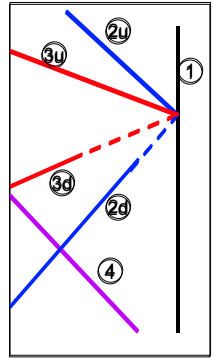

(b)

Fig. 4: Schematic diagram of tube-waves in the single-well survey: (a) Wave paths; (b) Arrivals on the seismogram.

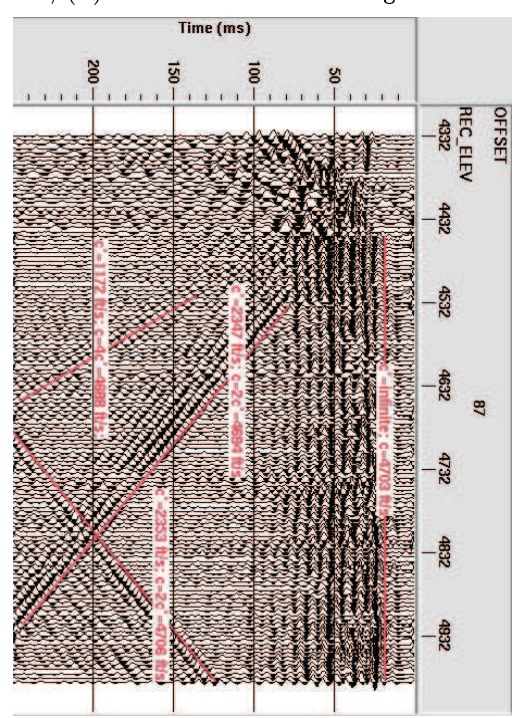

Fig. 5: Apparent velocity $\left(c^{\prime}\right)$ and true velocity $(c)$ of the direct, reflected, and multiple tube-waves in the field data with $\mathrm{AGC}$

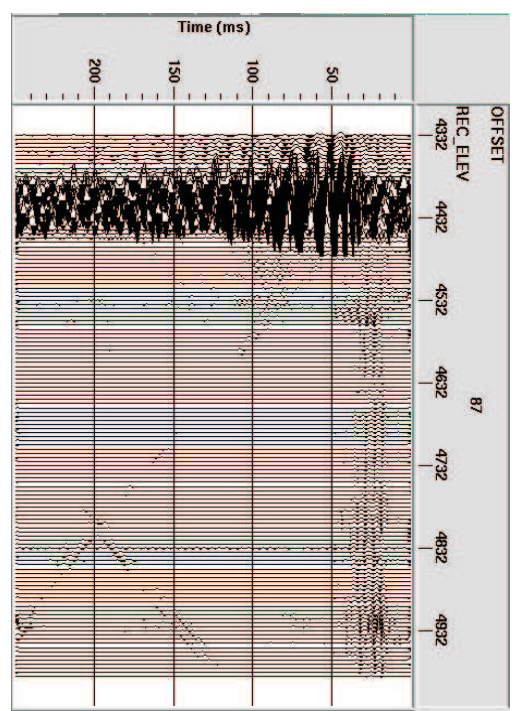

Fig. 6: True amplitude display of the data: the anomalous zone (4387-4442 ft) with low velocity and high amplitude.

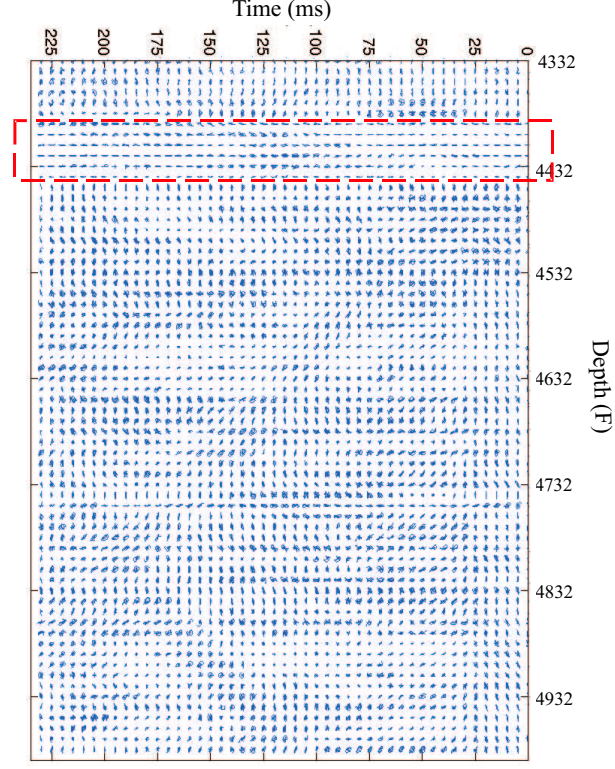

Fig. 7: Polarization diagram for receiver $\mathrm{H} 2$ and $\mathrm{V}$ of the source H2. The anomalous zone (in the red box) has horizontal polarization but the rest has vertical polarization.

the Menefee shale and is in the Cliffhouse sand. The predicted tube-wave velocity from logs (the 3 rd panel in figure $3 \mathrm{~b}$ ) for this zone is around $4700 \mathrm{ft} / \mathrm{s}$ which is much higher than the velocity of the observed direct wave on the field seismogram $(2900 \mathrm{ft} / \mathrm{s})$. Therefore formation properties obtained from logs do not explain the anomaly.

The particle motion study (Figure 7) shows the anomalous event is horizontally polarized, but the normal tubewave is vertically polarized. The horizontal polarization is consistent with flexural-waves, but the dispersion relation of flexural-waves (Paillet and Cheng, 1991) indicates that the velocity of the flexural-wave at the single-well frequency $(300-400 \mathrm{~Hz})$ can not be as low as that of the anomalous event in the field observations.

Comparing the polarization of the four common-offset gathers (offsets $87,97,107$ and $117 \mathrm{ft}$ ) by fixing the source and receiver depth, respectively, we find the anomaly only happens when the source is below $4300 \mathrm{ft}$ and the receiver is above $4442 \mathrm{ft}$. This indicates that it is not caused by either the source or receiver, but is related to some formation properties not explained by open-hole logs. Out of plane vertical gas-filled fractures can be one possibility, but more study on the amplitude of gas-filled fracture reflections are needed.

Another interesting finding is that the anomalous zone shows direct correspondence with the highest permeability zone interpreted from open hole logs. However, a mechanism which could explain the relationship is not yet determined.

\section{Modeling study}




\section{Single-well seismic data analysis and modeling}

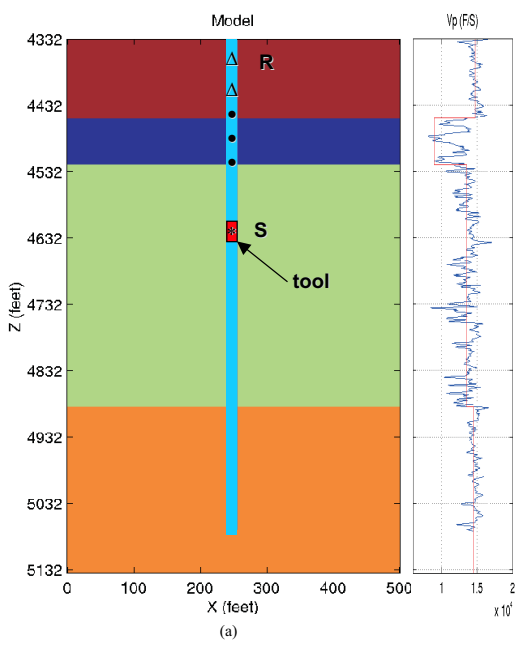

Fig. 8: (a) Model; (b) Blocked logs.

To aid the data analysis, modeling study using a 2-D variable-grid finite-difference parallel code is performed. Here we present one result to confirm the identified events in the field data.

Figure 8a shows the layered model with formation properties from the logs (figure $8 \mathrm{~b}$ ). The water-filled borehole is blocked at $5082 \mathrm{ft}$. A x direction dipole source, at 4622 $\mathrm{ft}$, is used to excite the model. A small rectangular steel box is placed around the source to represent the tool. Unlike the field survey with four receivers per shot, in the simulation, we put receivers on all grids along the borehole for one shot. The synthetic common shot gather with the whole depth coverage is used to assist the field data analysis since it is computationally costly to generate the same synthetic common offset gather as the field data. A variable grid mesh, which efficiently handles the small borehole in a large model, is used for the modeling.

The synthetic common shot gather, figure 9, shows tubewaves dominate the wavefield though $\mathrm{S}$-waves are visible in some traces. Direct tube-waves $(\mathrm{T}), \mathrm{C} / \mathrm{M}$ interface and well plug reflections $\left(T T_{i}\right.$ and $\left.T T_{p}\right)$, and multiple reflections between the tool and $\mathrm{C} / \mathrm{M}$ interface $\left(T T_{t} T_{i}\right)$ observed in the synthetic seismogram confirms the identified events in the field data.

\section{Conclusions}

We have conducted single-well acquisition, data analysis and modeling. The data analysis demonstrates that the consistently observable events in the field data are tubewaves. Except an anomalous zone with the low velocity and high amplitude, the direct, reflected and multiple tube-waves can be explained by the formation properties from logs and the survey geometry. Numerical modeling confirms our data analysis.

Future work will involves analyzing the events at the depths with fracture indications from FMI (Formation

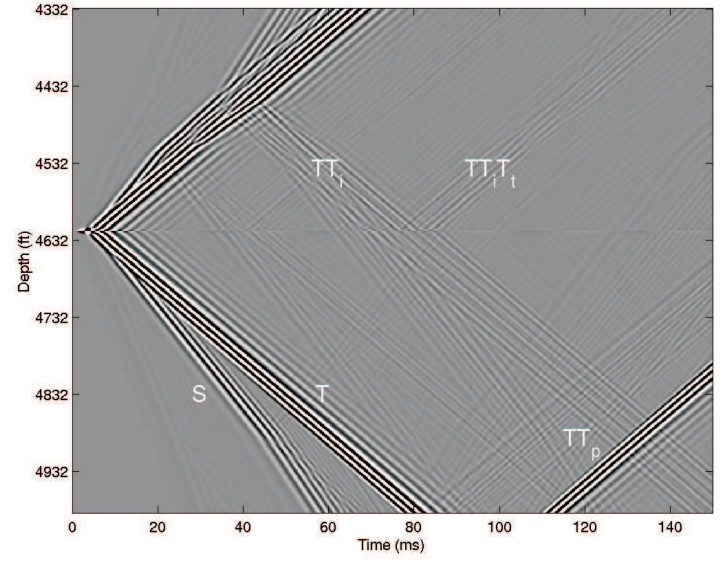

Fig. 9: Synthetic common-shot gather: $S$ - direct S-wave; $T$ - direct tube-wave; $T T_{i}-\mathrm{C} / \mathrm{M}$ interface reflected tube-wave; $T T_{p}$ well plug reflected tube-wave; $T T_{i} T_{t}$ - multiple tube-wave reflections between the tool and the interface.

\section{Micro Imager).}

\section{Acknowledgments}

Thank Richard T. Coates of Schlumberger-Doll Research for providing the well log data interpretation. This work was supported by the assistant Secretary for Fossil Energy, National Energy Technology Laboratory (NETL); LBNL processing was performed at the Center for Computational Seismology, supported by the Director, Office of Science, Office of Basic Energy Sciences, Division of Engineering and Geosciences, of the U.S. Department of Energy, both under contract No. DE-AC03-76SF000098.

\section{References}

Majer, E. L., Daley, T. M., Myer, L. R., Nihei, K. T. Queen, J., Sinton, J., Murphy, J., Fortuna, M., Lynn, H. B., Imhoff, M. A., and Wilson, R., 2001, San juan fracture characterization project: Status and current results: Lawrence Berkeley National Laboratory Report.

Majer, E. L., Queen, J. H., Daley, T. M., Murphy, J., Fortuna, M., Lynn, H., Coates, R., Eike, P., Sinton, J., Cox, D., Myer, L., Nihei, K., and Nakagowa, S., 2002, Scaling between logs, single well, VSP and surface seismic for fracture quantification in naturally fractured gas reservoirs: 72nd Ann. Internat. Mtg., Soc. Expl. Geophys., 2381-2384.

Paillet, F. L., and Cheng, C. H., 1991, Acoustic waves in boreholes: CRC Press.

White, J. E., 1983, Underground sound: application of seismic waves: Elsevier. 